\title{
Inferring Micro-Activities through Wearable Sensing for ADL recognition of Home-Care Patients
}

\author{
Mathangi Sridharan, John Bigham, Paul Michael Campbell, Chris Phillips, Member, IEEE, \\ and Eliane Bodanese, Member, IEEE
}

\begin{abstract}
In this study, we propose a novel context-based location aware algorithm for identification of low-level microactivities that can be used to derive complex Activities of Daily Living (ADL) performed by home-care patients. This is achieved by gathering location information of the target user using a wearable beacon embedded with magnetometer and inertial sensors. The shortcomings of beacon signal stability and mismatch issues in magnetic field sequences are overcome by adopting a hybrid three-phase approach for deducing the locus of micro-activities and their associated zones in a smart home environment. The suggested approach is assessed in two different test environments where the main intention is to map the location of a person performing an activity with pre-defined house landmarks and zones in the offline labelled database. In addition to recognition of low-level activities, the proposed method also identifies the person's walking trajectory within the same zone or between different zones of the house. Experimental results demonstrate that it is possible to achieve centimeter-level accuracy for recognition of micro-activities and a classification accuracy of $85 \%$ for trajectory prediction. These results are encouraging and imply that collection of accurate low-level information for ADL recognition is possible through integration of inertial sensors, Magnetic Field and Bluetooth Low Energy (BLE) technologies from the wearable without relying on other infrastructural sensors.
\end{abstract}

Index Terms-Activity Recognition, wearable sensing, locationbased context prediction, independent living, smart home.

\section{INTRODUCTION}

A DVANCEMENTS in cheap sensing technology and communication systems has helped improve quality of life by developing applications for various domains. There is an increasing demand for remote healthcare systems for oneperson households as it facilitates independent living in a smart home setting and does not require the presence of care-givers at all times. Routine activities such as eating, dressing, bathing, and toileting are commonly referred to as Activities of Daily Living ( $A D L)$, which people tend to do on a daily basis for normal self-care [1]. In most cases, the remote monitoring systems are developed to gather information to infer if the person has completed their routine activities on a daily basis. A common mode of sensing for ADL recognition is by detecting

Mathangi Sridharan, John Bigham, Paul Michael Campbell, Chris Phillips and Eliane Bodanese are with the School of Electronic Engineering and Computer Science, Queen Mary University of London, Mile End Rd, London E1 4NS, U.K. (email: m.sridharan@qmul.ac.uk; john.bigham@qmul.ac.uk; p.m.campbell@se16.qmul.ac.uk;chris.i.phillips@qmul.ac.uk;

eliane.bodanese@qmul.ac.uk). The author sequence stated in this paper follows the "first-last-author emphasis" norm.

Manuscript submitted November 2018. This work was supported in part by QMUL/Wellcome Trust Institutional Strategic Support Fund through the LSI Digital Health Award. human object interaction through ambient and infrastructural sensors. Additionally, collection of location information of the resident in their homes can assist in the ADL recognition process by providing context information and can help reduce the number of installed infrastructural sensors.

The Global Positioning System (GPS) that has been widely used for outdoor positioning, fails to provide adequate support for indoor localization due to the high attenuation of the satellite signal caused by roofs and walls inside buildings [2]. As a result, this has opened up research on alternative methods that are reliable and capable of achieving higher accuracy. Over the past few years, researchers have developed indoor positioning systems that are based on different mediums. Radio Frequency (RF) based systems such as Wi-Fi, Bluetooth, Radio Frequency Identification (RFID) and Ultra-Wide-Band (UWB) are the most popular technologies used in existing Indoor Positioning Systems (IPS) due to the widespread prevalence of hardware and existing networks [2]. Apart from these technologies, magnetic field based positioning systems have garnered extensive interest amongst the scientific community due to the fact that they are not prone to multi path degradation and can operate in obstructed Line of Sight (LOS) conditions [3],[4]. The anomalies in the ambient magnetic field, which are formed due to distortions caused by the surrounding structural steel elements, form the main basis for building magnetic maps for indoor localization [3],[5]. In comparison with Wi-Fi or Bluetooth Low Energy (BLE), the magnetic field has low sensitivity to changes in surrounding environment (movement of people or furniture) and therefore, has the capability to generate the same magnetic fingerprint over time [4]. Each of the above-discussed technologies has its share of limitations. UWB systems perform well in Non-Line-of-Sight (NLOS) conditions achieving a positioning accuracy of the order of $20 \mathrm{~cm}$. But most of these systems are commercially expensive when compared to other technologies and in some cases, the signals may be blocked by large metallic objects [6]. The decrease in accuracy of Wi-Fi based positioning caused by user movement and presence of obstructions remains a main concern [7]. Zhao et al. compared the positioning accuracy of BLE and Wi-Fi in a similar test bed environment and the results showed that BLE outperformed Wi-Fi by around 27 percent [8]. Having considered the advantages and disadvantages of both of these technologies, we decided to opt for a BLE based inverse beacon positioning method in our study due to its inherent benefits. However, beacon signals that are mainly suited for proximity based positioning are often erratic and do not contribute to fine-grained positioning when used 
as a solo solution [9]. BLE is known to work best when combined with other technologies. Similarly, magnetic field matching, when used as an independent IPS solution, may not be enough for accurate positioning as only three parameters are considered and hence increases the probability of having the same fingerprint in different locations [7],[9]. Therefore, an extensive reference database consisting of continuous samples of magnetic signatures is required to improve the accuracy and uniqueness of the mapping. Prior research suggests that implementing hybrid technologies for indoor localization has proved advantageous since they exhibit sufficient positioning accuracy [10],[11]. In this study, we propose a novel algorithm for low-level ADL recognition (also referred to as microactivities) using only a wearable embedded with beacon and magnetometer to find the locus of the target user and track their movement between different zones of the house. This is achieved by the collaborative use of BLE technology with inertial sensors to reduce the search space for magnetic field sequence matching. We perform different assessments and a case study in order to verify the hypothesis that a standalone hybrid implementation of the above stated technologies can provide good recognition accuracy when a high number of micro-activities and user trajectories are considered for classification.

The main contribution of this paper is two-fold.

- We propose a novel algorithm using a wearable for near real-time recognition of low-level micro-activities and their associated zone of occurrence within the house. The resulting outcome helps in providing useful location context information for discovery of complex ADL's. This method is particularly useful in developing monitoring applications for home-care patients and also for sheltered accommodation, which are typically studio or onebedroom apartments. In order to demonstrate and verify the accuracy of the proposed system, extensive experiments are conducted in two different home environments under strong Non-Line of Sight (NLOS) conditions.

- In addition to recognizing the micro-activities, the proposed algorithm is capable of predicting accurately the movement of a person between different landmarks within a room and also between different zones of a house without relying on data from other infrastructure-based sensors.

This location aware system has been developed as part of a larger project for monitoring depression patients at home. The measured output data of the positioning system will be integrated to our complex activity recognition system, where the main objective is to sustain a minimal sensing environment at home without compromising on the accuracy of the predicted activities. Furthermore, the resulting location information can also be used in segmentation of raw sensor data stream from ambient sensors for differentiation between individual activities.

The remainder of this paper is organized as follows. Section II presents a survey of the related work. The necessary background and definition concepts are explained in Section III. A brief overview of the system architecture is presented in Section IV, followed by the description of the algorithm in Section V. The evaluation results investigated in the experimental test-bed using the proposed approach are presented in Section VI. In Section VII, we analyze and discuss the findings of several aspects of the proposed method. Section VIII concludes the paper.

\section{RELATED WORK}

Different sensor technologies have been used for carrying out activity recognition. Some works such as [12],[13] rely on smartphones for monitoring activities. The authors of [12] use just a single smart phone to identify simple and complex activities. Their experiments yielded a classification accuracy of above $90 \%$ for simple activities, but drops to $50 \%$ for complex activities. Using smart phones is unrealistic for patient or elderly monitoring applications as it forces the resident to carry their bulky phones with them at all times. Other works depend on object-based sensing methods for recognizing different activities [14],[15]. Instrumenting most of the daily-use objects with sensors increases the deployment cost and is not a practical solution for a home environment. Computer Vision-based systems such as RGB cameras, depth sensors and infrared sensors have also been a popular choice for tracking a person in an indoor environment [16]. However, these solutions were identified as too intrusive in a smart home environment according to a recent study [17]. The results showed that camera based medical monitoring inside a home had the lowest acceptance rate at $13.46 \%$.

An overview of recent work on ADL recognition systems, where location information is used for identification of lowlevel or complex activities, is presented here. Torres et al carried out experiments with a smart phone in seven different urban flats to study whether the Wi-Fi fingerprinting approach is feasible for in-home monitoring applications [13]. Their results provided an average accuracy of $89 \%$. In [18] and [19], the authors implemented a BLE fingerprinting approach using beacons for monitoring of nursing home residents and for detecting frailty in older adults based on indoor localization habits, respectively. However, all these systems were designed to predict simple activities based on the user's location with just room-level accuracy. Other studies have used location as one of the parameters alongside other sensing methods to predict multiple complex ADL's [15][20]. The authors of [20] use positioning sensors along with power meters for real time recognition of routine activities in a smart home. The system achieves an overall classification accuracy of $79.39 \%$. In [15], multiple events from binary sensors, a capacitive smart floor and a wearable beacon with an accelerometer are combined together in a real-time segmentation-free approach to predict 24 different personal activities in an apartment. Such systems predict a high number of ADLs, but increase the deployment cost and sensing complexity. Failure to track user movement between different zones of the house is also a major drawback in the above-discussed solutions. This may provide inconsistent results due to missing sensor readings in real or near real-time recognition systems. We prefer to have a system in place that makes use of low-cost sensing equipment 
and is still able to provide adequate accuracy when predicting more than ten activities.

Locating a person inside a home requires contextual and useful information related to their inherent surroundings, which can be mapped easily to an activity recognition framework. Using a global coordinate system for location mapping inside a house for such applications is impractical. The Received Signal Strength Indicator (RSSI) collection process during the training stage is much less strenuous when a sequence of RSSI samples can be collected along a known pathway as opposed to dividing the house floor plan into a grid like pattern and collecting data over random discrete points. Prior research suggests that position estimation using sequential RSSI values along a path is less variant when compared to point based prediction methods [21]-[23]. RF fingerprinting performed in this way is known to be more resilient to changes in the surrounding environment because the sequential values collected along a pathway have a distinct signature and consequently, this approach is more suitable for beacon fingerprinting to account for its high signal variability. The authors of [21] and [23] have used sequences of RSSI data for radio map creation, but importance has not been given to the inclusion of routes that reflect a person's daily routine. It is rather helpful to create a system, which integrates various elements of a home such as couch, dining area, sink, stove, bed and so on into the fingerprint collection database. The focus of our developed system is not in finding the $(\mathrm{x}, \mathrm{y})$ coordinates of the target, but determining whether the target is stationary at certain defined locations of interest, e.g., sitting on the couch or in the dining area, or moving along certain defined paths, e.g., dining to kitchen area, bedroom to bathroom and so on. Previous work focussed on a hybrid two-phase approach for recognition of landmarks and routes in a complex indoor environment using a wearable and a smartphone [22]. The current study builds on our prior work using a single wearable coupled with inertial sensing to infer context from location assessed in two different trial homes.

\section{BACKGROUND AND DEFINITIONS}

In this study, we refer to micro-activities as a combination of low-level activities derived from location context that maybe part of a high-level complex activity such as preparing breakfast, watching TV and so on. The study also focuses on recognizing the walking routes to account for the transition when a person moves between different landmarks within the house. The walking routes have been further categorized as short routes (less than 5 steps) and long routes (greater than 5 steps) based on the average number of steps a person takes in order to overcome the issue of overlapping routes in a given space. We present below the background, along with the definition of several important concepts.

\section{A. Inverse Beacon Positioning}

Most of the existing positioning systems employ a set of fixed beacons at known locations and a moving receiver such as a mobile phone. However, using multiple receivers at fixed positions to track a moving beacon is better suited for use cases such as monitoring a person or asset tracking [24]. This type of technique is commonly referred to as the Inverse Beacon Positioning method. The implementation is based on the principle that the RSSI signal decays with the increasing distance between the beacon and the receiver.

Definition 1. A beacon RSSI profile $\left(\overrightarrow{R S S I}_{i}\right)$ is a vector of individual RSSI values observed at position $\overrightarrow{L_{i}}$ from $n$ nearby receivers. $\overrightarrow{L_{i}}=\left(d_{x}^{i}, d_{y}^{i}\right)$ corresponds to a specific point marked by its two-dimensional Cartesian label. In this study, we use a set of Raspberry-Pis as the receiver of the beacon signal. The RSSI vector measured at any given point is represented by:

$$
\overrightarrow{R S S I}_{i}=\left\{P i_{R S S_{1}}, \ldots, P i_{R S S_{n}}\right\}
$$

where $P i_{R S S_{1}}, \ldots, P i_{R S S_{n}}$ are the individual signal strength of the Raspberry-Pi receivers.

Definition 2. The beacon RSSI fingerprint database $\left(R S S I_{D B}\right)$ is modeled as:

$$
R S S I_{D B}=\left\{R S S_{S_{1}}, \ldots, R S S_{S_{K}}\right\}(1 \leq k \leq K)
$$

where $R S S_{S_{k}}$ represents the collective beacon fingerprint measurement for an individual micro-activity or walking route identified by $S_{k}(1 \leq k \leq K)$ with $K$ being the total number of micro-activities and routes in the database. For any given $S_{k}, N$ training samples are present where each sample is an individual fingerprint $B_{i}$.

A beacon RSSI fingerprint $\left(B_{i}\right)$ is a combination of RSSI profile $\overrightarrow{R S S I}_{i}$ measured at location $\vec{L}_{i}$, labeled by its corresponding route or micro-activity $S_{k}$. It is represented by:

$$
B_{i}=\left[\vec{L}_{i}, \overrightarrow{R S S I}_{i}, S_{k}\right](1 \leq i \leq N)
$$

For cases where $S_{k}$ is a walking route, the training examples will be a sequence of RSSI values recorded at their corresponding position co-ordinates along the considered walking path. The database may contain duplicate values of $\overrightarrow{L_{i}}$ as several RSSI readings are captured at the same position. Sample data of the training database $\left(R S S I_{D B}\right)$ is presented in Table I.

TABLE I

RSSI TRAINING DATABASE SAMPLE

\begin{tabular}{|ccccccccccc|}
\hline $\mathbf{X}$ & $\mathbf{Y}$ & $\mathbf{P i}_{\text {RSS1 }}$ & $\mathbf{P i}_{\text {RSs2 }}$ & $\mathbf{P i}_{\text {RSs3 }}$ & $\mathbf{P i}_{\text {RSS4 }}$ & $\mathbf{P i}_{\text {RSss }}$ & $\mathbf{P i}_{\text {RSs6 }}$ & $\mathbf{P i}_{\text {RSs7 }}$ & $\mathbf{P i}_{\text {RSss }}$ & Micro-Activity/Route \\
\hline 5.296 & -0.647 & -60 & -79 & -86 & -75 & -76 & -81 & -74 & -82 & Main Door to Bedroom Door \\
5.296 & -0.647 & -57 & -75 & -85 & -69 & -84 & -89 & -76 & -79 & Main Door to Bedroom Door \\
5.296 & -0.647 & -55 & -74 & -79 & -72 & -77 & -85 & -79 & -82 & Main Door to Bedroom Door \\
1.811 & 1.046 & -72 & -84 & -81 & -90 & -74 & -78 & -81 & -76 & Couch to Main Door \\
1.811 & 1.046 & -78 & -89 & -83 & -91 & -69 & -78 & -84 & -77 & Couch to Main Door \\
1.811 & 1.046 & -70 & -85 & -84 & -92 & -69 & -82 & -87 & -92 & Couch to Main Door \\
\hline
\end{tabular}

\section{B. Magnetic Field Vector (MFV) Sequence Matching}

Definition 3. The Magnetic Field Vector, $\overrightarrow{M F V}_{i}$ profile represents the magnetic field strength in $\mathrm{x}, \mathrm{y}, \mathrm{z}$ direction observed at position co-ordinates $\overrightarrow{L_{i}}=\left(d_{x}^{i}, d_{y}^{i}\right)$. It is represented by:

$$
\overrightarrow{M F V}_{i}=\left\{M F V_{x}, M F V_{y}, M F V_{z}\right\}
$$

Definition 4. Similar to $R S S I_{D B}$, the MFV training database $\left(M F V_{D B}\right)$ is populated with sequential three-dimensional vector readings modeled as:

$$
M F V_{D B}=\left\{M V_{S_{1}}, \ldots, M V_{S_{K}}\right\}(1 \leq k \leq K)
$$


where $M V_{S_{k}}$ represents the collective magnetic field measurement for each micro-activity or walking route identified previously as $S_{k}(1 \leq k \leq K)$. Each MFV fingerprint $\left(M_{i}\right)$ observed at position co-ordinates $\overrightarrow{L_{i}}=\left(d_{x}^{i}, d_{y}^{i}\right)$ for a given micro-activity or route $S_{k}$ is represented by:

$$
M_{i}=\left[\vec{L}_{i}, \overrightarrow{M F V}_{i}, S_{k}\right](1 \leq i \leq N)
$$

where $N$ is the number of training samples observed for individual $S_{k}$.

Definition 5. The Dynamic Time Warping (DTW) technique is used as the distance measure to compute the similarity between two MFV sequences. DTW is a well-known algorithm to measure the similarity between two sequential series of different lengths that vary in time or speed. It has widespread application in image processing, speech recognition, data mining, robotics, manufacturing and other classification techniques [25]. The DTW distance measure has an innate advantage over Euclidean distance for time series measurement as the latter fails to provide a correct measure when there are small distortions in the time axis. DTW is more robust for comparing MFV sequences as it allows a one to many mapping (compression and stretching the time axis) of one or both the sequences to obtain a suitable alignment. An iterative procedure is performed for all the eligible routes, which involves constructing a matrix between the offline and measured magnetic sequence to find the warping path that minimizes the overall cost function. This is calculated using the dynamic programming approach given by $\mathrm{Eq}(7)$.

$$
\begin{aligned}
\operatorname{DTW}(n, m)= & d\left(q_{n}, c_{m}\right)+ \\
& \min \{\operatorname{DTW}(n-1, m-1), \\
& \operatorname{DTW}(n-1, m), \operatorname{DTW}(n, m-1)\}
\end{aligned}
$$

where $d\left(q_{n}, c_{m}\right)=(q[n]-c[m])^{2}$ is the squared distance between the sample points. $Q=\left\{q_{1}, q_{2} \ldots q_{N}\right\}$ and $C=$ $\left\{c_{1}, c_{2} \ldots c_{M}\right\}$ are the online and offline magnetic sequences of length $N$ and $M$, respectively. Squared Euclidean distance is selected to calculate the cost measure in $\operatorname{Eq}(7)$ as it provides better accuracy in comparison with Euclidean or Manhattan distance measures. The final predicted route is the one with the least distance measure, which implies a high similarity between the measured sequences.

Definition 6. $\overrightarrow{R S S I}_{\text {Online }}$ and $\overrightarrow{M F V}_{\text {Online }}$ are the respective online beacon and magnetic sequences collected using the wearable for prediction of location context of the target user using the reference $R S S I_{D B}$ and $M F V_{D B}$. The task is to find the label $S_{K}$ using the algorithm described in Section V.

\section{SySTEM OVERVIEW}

\section{A. Hardware Requirements}

The overall system architecture is composed of a set of Raspberry-Pis that function as receivers and a transmitting wearable beacon with embedded sensors known as MetaMotionR manufactured by MbientLab [26]. The wearable allows on-chip logging or streaming of sensor data and it comes enclosed in a waterproof casing with a rubber clip. Amongst the multiple onboard sensors present in the wearable, we use the magnetometer, the built-in step detector module and the beacon functionality. Raspberry Pi 2 Model B devices were used in Trial home-1 and Raspberry Pi 3 Model B devices were used in Trial home-2 as receivers of the beacon signal. The number of Raspberry-Pis to be deployed in a specific household is decided such that every part of the house falls within the coverage area of at least two Raspberry-Pis and their placement is decided based on the activities to be monitored. The coverage was roughly estimated by taking into account the number of Raspberry-Pis detecting the beacon for any given route or micro-activity [27]. Accuracy is improved with increasing numbers of receivers detecting the beacon.

\section{B. Design and Implementation}

One of the Raspberry-Pis is programmed to be the master and the remaining devices function as slaves. All RaspberryPis collect beacon RSSI data along with the timestamp. The master Raspberry-Pi receives the raw data from the slaves every few seconds and combines it into a single file. Linear interpolation is performed in case of missing RSSI readings per second. The magnetometer sensor readings and step counter data with the corresponding timestamp are simultaneously transmitted from the wearable to the master receiver. The final processed data consisting of the beacon RSSI, magnetometer and step detector readings are used as input for Algorithm 1.

\section{Battery Life and Power Consumption}

The MetaMotionR wearable is powered by a rechargeable $100 \mathrm{mAH}$ lithium-ion $3.7 \mathrm{~V}$ battery that can last for about 1.5 weeks on a single battery charge. Other wearables from MbientLab that use a coin cell battery can also be worn that may be more suitable for elderly monitoring applications, in which case the battery can last up to 1 year. The power consumption of the MetaMotionR wearable ranges from $20 \mu \mathrm{A}$ to $20 \mathrm{~mA} @ 3.0 \mathrm{~V}$. In this study, the sampling frequency of the beacon is set at $10 \mathrm{~Hz}\left(f_{s 1}=10 \mathrm{~Hz}\right)$ and the magnetometer sampling frequency is set at $10 \mathrm{~Hz}\left(f_{s 2}=10 \mathrm{~Hz}\right)$, such that the battery life of the wearable is effectively prolonged while maintaining sufficient accuracy.

\section{Data Collection Process: Beacon RSSI, Magnetometer and Step Counter data}

The wearable beacon is clipped on to the shirt collar such that it is positioned at chest level at all times. The training and the test data were collected over a ten-day period in Trial home-1 and over a week in Trial home-2. The measurements were made on different days and at different times of the day. A number of walking routes and micro-activities were chosen to reflect regular domestic human behavior to provide the most realistic scenario for testing. The RSSI and MFV samples were collected for conditions when a person is performing an activity with minimal movement (e.g., sitting in the dining area or couch) and when a person walks along a certain path both in the forward and reverse directions (e.g., bedroom to bathroom, bathroom to bedroom). Due to the nature of 
the classification technique used during the training stage, sequences of continuous readings of RSSI and MFV are considered in this study instead of point-to-point estimation. For the radio map construction, the sequences were measured for 30 s at each position along a walking route with step size measuring approximately $50 \mathrm{~cm}$. The corresponding timestamp and route/position label were recorded using a mobile application for annotation developed by us. All the routes present in the training database are classified as either MicroActivities/Short Routes or Long Routes based on the resulting step count measured using the built-in step detector module in the wearable. The training database size and overhead in this case is considerably less when compared to other existing data collection methods as limited samples are amassed for a short duration of $30 \mathrm{~s}$, making the calibration stage simple. The ground truth of the collected test data in terms of position co-ordinates for cross-validation were measured using a laser distance meter and recorded using the developed annotation application for accuracy calculation in both the trial homes.

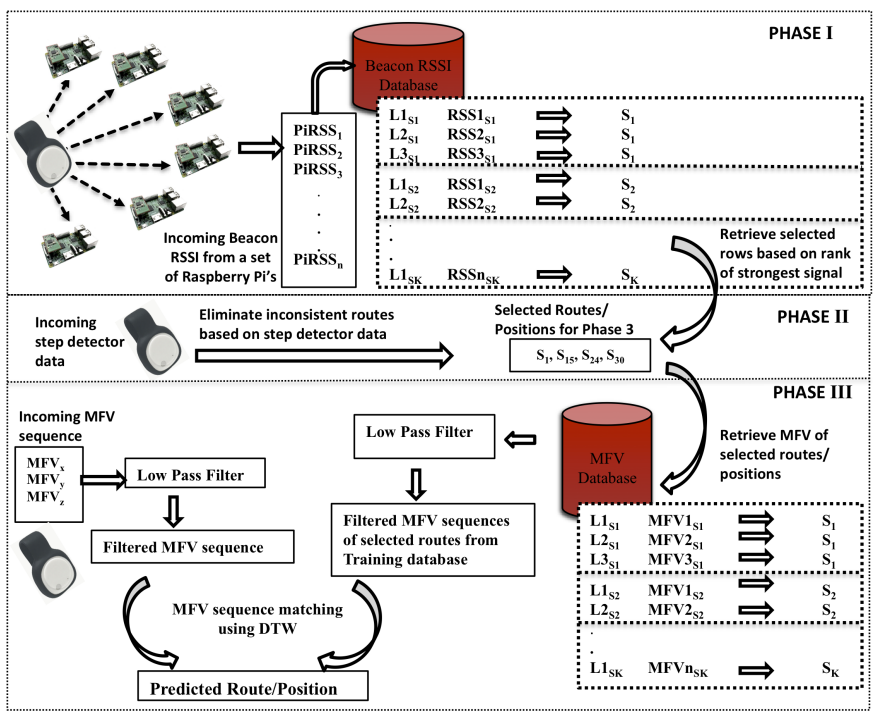

Fig. 1. Schematic Diagram of the Proposed System

\section{Algorithm For Detection Of Micro-Activities AND WALKING ROUTES}

The proposed algorithm constitutes three main phases for detection of low-level activities and walking routes. It follows a data-driven approach as the method relies on the training data collected using the wearable and their respective ground truth labels expressed in terms of landmarks and routes within an indoor environment. Fig. 1 illustrates the overall system architecture. A brief description of the three phases involved in the positioning algorithm is given below and their respective pseudocode is shown in Algorithm 1.

\section{A. Description of the Positioning Algorithm}

a) Phase I - Route Selection Using Beacon RSSI Fingerprinting: The offline collected RSSI training samples and the online measured sequences are initially sorted based on their strongest beacon signal strengths. An individual rank matrix
Algorithm 1 Route Prediction and Detection of MicroActivities

Inputs: $\overrightarrow{R S S I}_{\text {Online }}$ and $\overrightarrow{M F V}_{\text {Online }}$, stepCount

Output: $R \leftarrow$ Predicted Walking Route or micro-activity

1: for $\mathrm{i}=1$ : $\operatorname{sizeof}\left(R S S I_{D B}\right)$ do

2: $\quad A \leftarrow$ Sort $\overrightarrow{R S S I}_{i}$ in descending order

3: end for

4: for $\mathrm{i}=1$ : sizeof $\left(R S S I_{\text {Online }}\right)$ do

5: $\quad B[i] \leftarrow$ Get corresponding label $S_{k}$ for matched $\overrightarrow{R S S I}_{i}$ in $R S S I_{D B}$ after sorting in descending order

6: $\quad$ occ $[i] \leftarrow$ Compute frequency of occurrence of matched $S_{k}$ in $B[i]$

7: end for

8: $C \leftarrow$ Sort $B$ based on Sum(occ), groupCount incase of ties, where groupCount $=$ no. of routes grouped by occ

9: $D \leftarrow$ Retrieve top 10 or less $S_{k}$ from $C$

10: if stepCount $\geq 0 \& \&$ stepCount $\leq 5$ then

11: selected $S_{k} \leftarrow$ All Micro-activities \& Short Routes applicable in $D$

12: else

13: $\quad$ selected $S_{k} \leftarrow$ All Long Routes applicable in $D$

14: end if

15: $M F V_{\text {Train }} \leftarrow \overrightarrow{M F V}_{i}$ of selected $S_{k}$ from $M F V_{D B}$

16: for $\mathrm{i}=1$ : sizeof $\left(M F V_{\text {Train }}\right)$ do

17: $\quad R \leftarrow$ findBest $S_{k}\left(\overrightarrow{M F V}_{\text {Train }}(i), \overrightarrow{M F V}_{\text {Online }}\right)$ //Predict $S_{k}$ label using $D T W$

18: end for

19: return $R$

for the training and online data is then created, where each row contains the Raspberry-Pi identifier arranged in order of the strongest signal. Both these ranked datasets are compared against each other to find similarities between them and a list of corresponding matched $S_{k}$ labels are returned. The routes and positions for the next phase are selected based on the total number of occurrences of matched labels $(\operatorname{Sum}(o c c))$ and frequency of occurrence for each position co-ordinate over the entire length of the measured test sequence (groupCount). The number of highest ranked positions/routes considered for Phase II depends on the accuracy of the Beacon RSSI fingerprinting method. An optimal count is chosen such that the correct position/route is part of the magnetic search area in the next phase. In this study, we have set the maximum count to ten or less for selection from the resulting output of Phase I.

b) Phase II - Elimination of Inconsistent Routes from Phase I Using the Step Detector Module: Data from the step counter is used to identify if the selected routes from Phase I fall under Type I (Micro-Activities and Short routes $(\leq 5$ steps)) or TypeII (Long routes (> 5 steps)) category. Based on this classification, extraneous routes are excluded and only the relevant routes are carried forward to the next phase. Micro-activities and short routes have been grouped together as there is a likelihood that the step detector may produce false positives or false negatives when small strides between 'kettle to sink' or 'fridge to microwave' are considered. This 


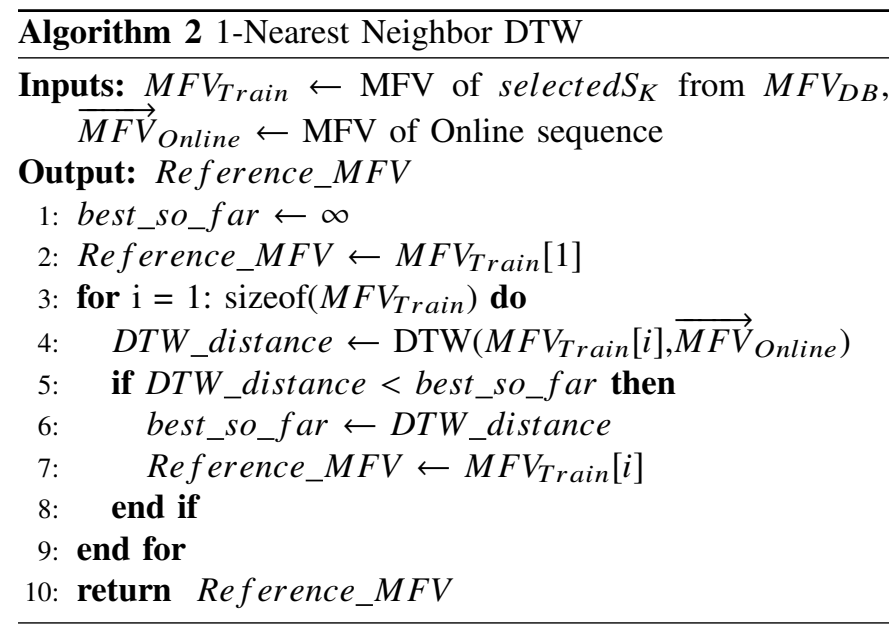

is especially true in a kitchen scenario when the objects of interest such as kettle, stove, sink, microwave are very close to each other and transitional changes between two stationary positions near different objects/landmarks need to be taken into consideration. Inclusion of the step counter assists in improving the accuracy by easily eliminating the overlapping routes in an indoor space. For example, Couch to Dining is a short route that overlaps with the longer route Couch to Main Door. Results from the step detector help in differentiating and choosing the applicable route for that particular instance. It is also beneficial for the next phase as it further reduces the magnetic matching searching space. This helps in improving the overall time complexity since the DTW technique that is used in the next phase is known to be a computationally expensive algorithm. Based on the requirement, the step detector can be configured in 3 modes: Normal/ Sensitive/ Robust.

c) Phase III - Magnetic Matching (MM) using DTW:

The magnetic signatures of the selected routes from Phase II are alone considered from the MFV training database and consequently, the overall efficiency is improved as the search space for MFV fingerprinting is significantly reduced. We apply a low pass filter to smooth the noise of the magnetometer sensor data before calculating the similarity measure. Nearest Neighbor Dynamic Time Warping algorithm that makes use of the dynamic programming approach given by $\mathrm{Eq}(7)$ is used to compute the similarity between two MFV sequences. The pseudocode for the 1-Nearest Neighbor DTW is presented in Algorithm 2.

\section{EXPERIMENTAL STUDY}

\section{A. Layout of the Test-Beds}

The suggested approach is assessed in two different test environments where the main aim is to map the target's location with the pre-existing routes and positions in the reference database. Two different individuals in their respective flats carried out the experimental study. Both the test-beds chosen are one-bedroom apartments, as we focussed our study to find ways to effectively distinguish activities based on location information when a person moves between various landmarks in a small space. Fig. 2(a) and Fig. 2(b) illustrate

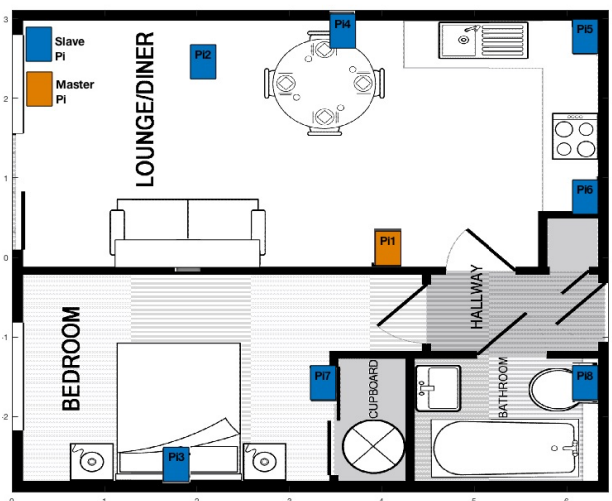

(a) Trial Home-1

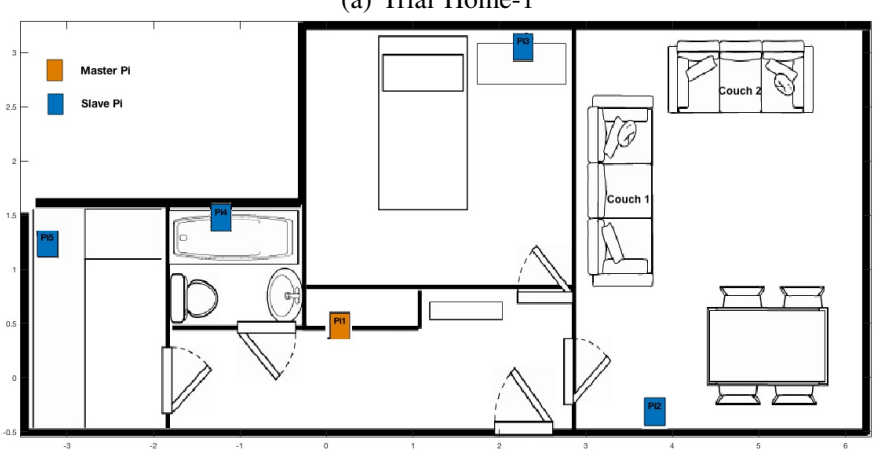

(b) Trial Home-2

Fig. 2. Trial Home Layout

the layout of Trial home-1 $(6.45 \mathrm{~m} \times 6 \mathrm{~m})$ and Trial home- 2 $(10.57 \mathrm{~m} \times 4.44 \mathrm{~m})$, respectively, along with the placement of the master and the slave Raspberry-Pis. To provide comprehensive Bluetooth coverage of the entire house, a total of eight Raspberry-Pi receivers were deployed in Trial home-1, while five Raspberry-Pi receivers equipped with either $1 \mathrm{dBi}$ or $5 \mathrm{dBi}$ external antennas were placed in Trial home-2. The reason for testing with two different antennas in the 2 nd trial home was to investigate if an increased antenna gain helps in maintaining the same level of accuracy with reduced number of RaspberryPi receivers. $R S S I_{D B}$ and $M F V_{D B}$ contain training samples for a total of 40 micro-activities and walking routes in Trial home- 1 and a total of 36 routes and micro-activities for Trial home-2.

\section{B. Performance Assessment Of Recognition Of Micro- Activities in Trial home-1}

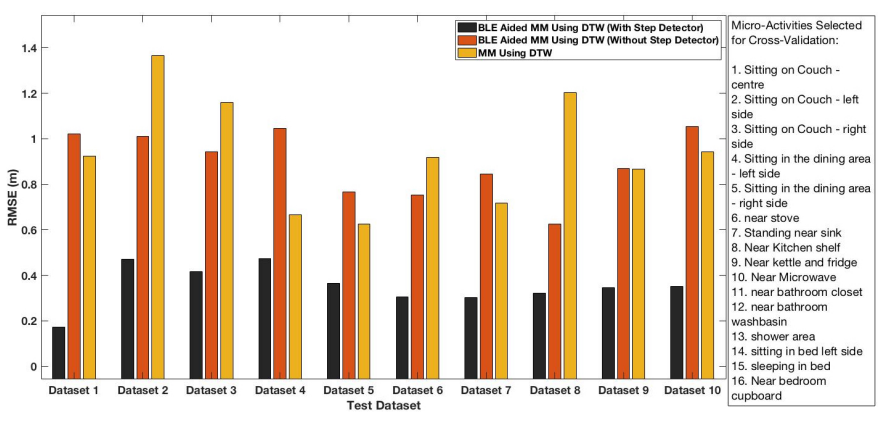

Fig. 3. Average RMSE measure for recognition of micro-activities 


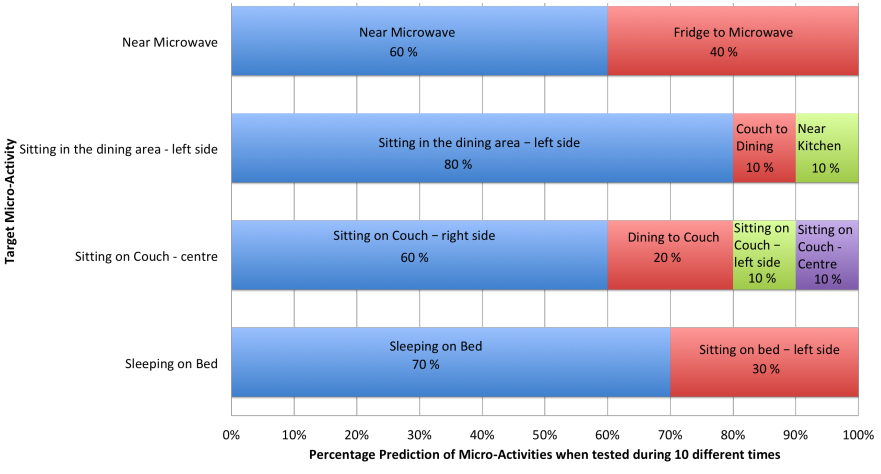

Fig. 4. Performance assessment of individual micro-activities

a) Error Calculation: Test samples for cross-validation were collected for a sub-section of routes/positions measured multiple times over different days within the trial home. The Root Mean Square Error (RMSE) was used as the performance measure for determining the standard deviation of the predicted locus of the micro-activities against the position coordinates of the ground truth. Fig. 3 illustrates the performance comparison of average RMSE computed for ten different test datasets for 16 micro-activities using three approaches - BLE aided MM using DTW with step detector (BLE-MMDTW), BLE-MMDTW (without step detector) and MM using DTW (MMDTW). The proposed algorithm using the hybrid BLEMMDTW method that includes the step counter had the least RMSE measure in each of the ten test cases with an overall average RMSE of $0.55 \mathrm{~m}$. The average RMSE for the BLEMMDTW (without step detector) and the MMDTW methods were $1.09 \mathrm{~m}$ and $1.13 \mathrm{~m}$, respectively. RMSE was chosen as the evaluation metric over classification accuracy in this scenario as we were interested in measuring the degree of closeness of the false positive results with the ground truth. For instance, the classification results using ten different test datasets for micro-activities: Sleeping on bed, Sitting on Couch - centre, Sitting in the dining area - left side and Near microwave shown in Fig. 4 indicate that the activities predicted as false positives are very close to the ground truth and the predicted outcome in each case was associated with the actual microactivity taking place. In Fig. 4, the micro-activity Sitting on Couch - centre was classified six times as Sitting on Couch right side, twice as Dining to Couch and one each for Sitting on Couch - left side and Sitting on Couch - centre when tested at ten different instances. However, the predicted outcome in each case refers to Couch as the main landmark where the activity is carried out. Similarly for the sleeping activity, the classification labels Sleeping on bed and Sitting on bed - left side maybe different, but the locus of both these activities are almost identical (Refer Fig. 4). The same can be said for the predicted outcome of other micro-activities. It must be noted that the false positive results occur as multiple positions and routes are considered for classification in this study (40 positions and routes). Sub-meter accuracy results demonstrate the merit of the approach especially given the high number of different micro-activities that are classified (16 activities excluding routes).This form of location context information is crucial for supporting complex ADL recognition when the number of physical sensors deployed in the smart home is small. In other words, it is possible for us to maintain a minimal sensing environment and recognize low-level activities by using just the wearable as the lone sensor data.

b) Zone Based Classification Accuracy Of MicroActivities: In order to check the room level accuracy of the detected micro-activities, Trial home-1 was divided into

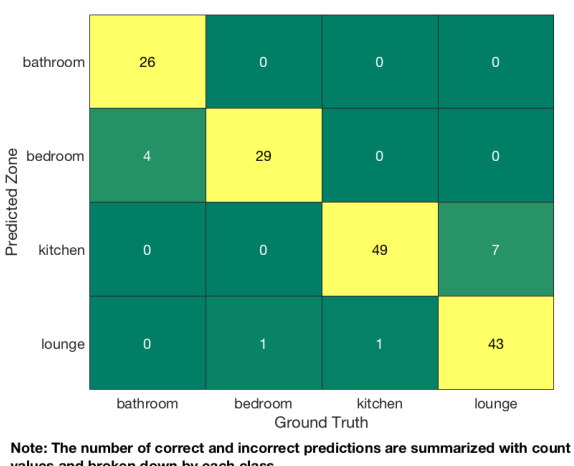

Note: The number of correct and incorrect predictions are summarized with com
values and broken down by each class.
Classification accuracy = Correct predictions $/$ Total predictions * $100=91.87 \%$

Fig. 5. Confusion matrix for Zone-based recognition of Micro-Activities

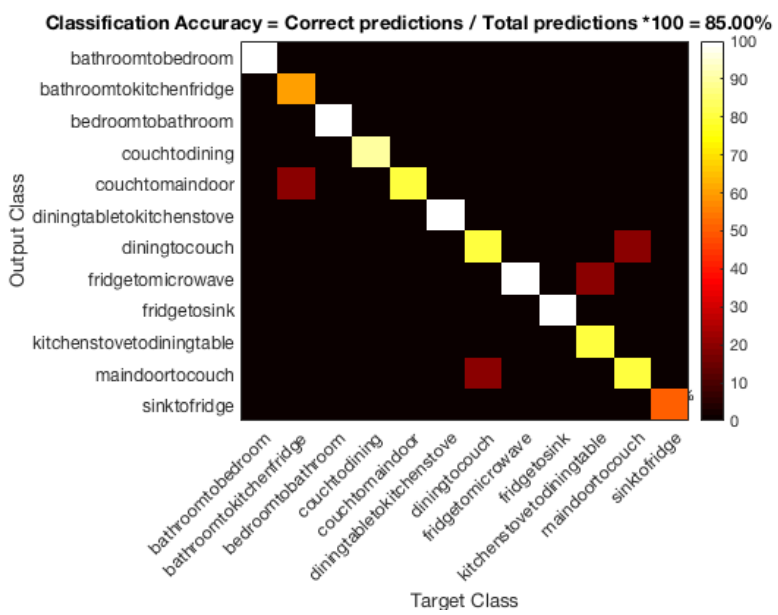

(a) BLE-MMDTW Method

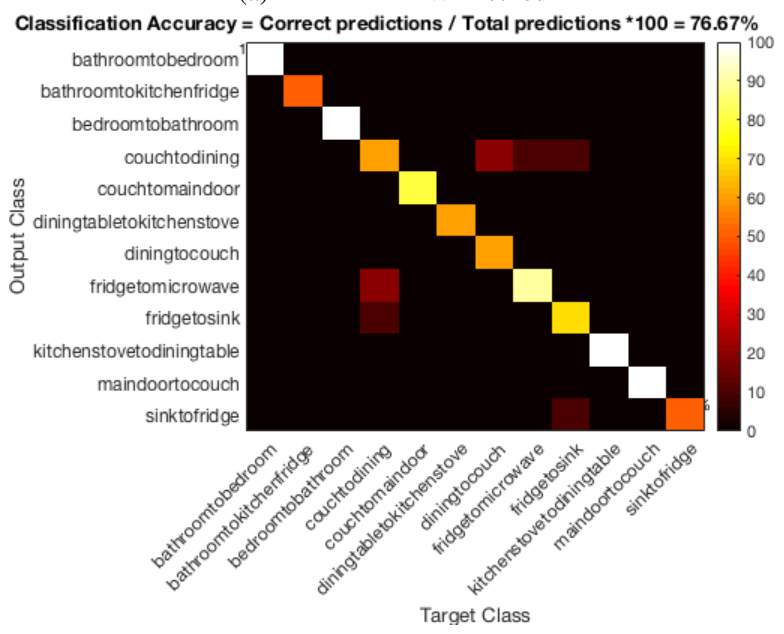

(b) MMDTW Method

Fig. 6. Recognition confusion matrix for walking routes 


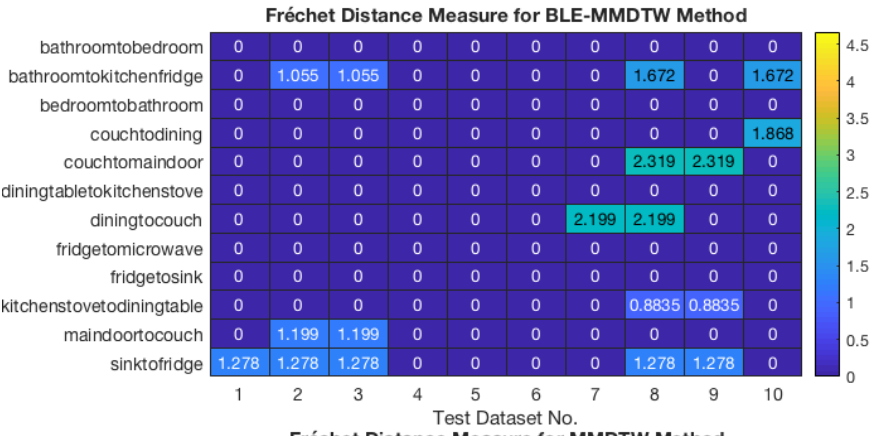

Fréchet Distance Measure for MMDTW Method

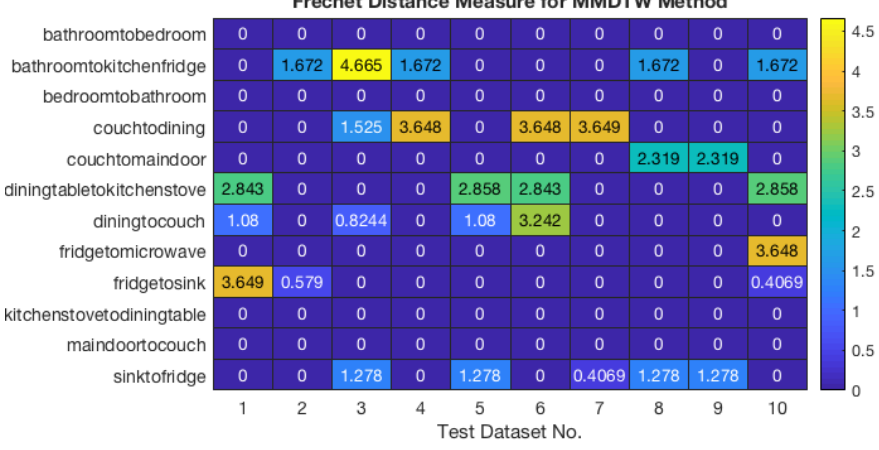

Fig. 7. Performance comparison of walking routes using Fréchet Distance in Trial home-1

five zones namely: Lounge, Bedroom, Bathroom, Kitchen and Hallway. Based on the earlier cross-validation results using the ten test datasets, the 16 micro-activities were classified into their respective zones in the trial home. The confusion matrix for zone-based recognition of micro-activities using the proposed BLE-MMDTW method is shown in Fig. 5. A classification accuracy of $91.87 \%$ was achieved, indicating that the zone of occurrence of the micro-activities was recognized correctly in most cases inside a confined living space. The classification accuracy in this study was obtained by calculating the percentage ratio of correct predictions to total predictions made. However, the classification accuracy drops to $51.25 \%$ when the beacon data was eliminated and the MM approach was carried out independently.

\section{Performance Assessment Of Recognition Of Walking Routes in Trial home-1}

Apart from the detection of micro-activities and their zone of occurrence, the proposed algorithm also tracks the walking trajectories of the target user. This helps to monitor the movement of the user between different rooms of the house. Ten different test datasets for 12 walking routes were collected for evaluating the classification efficiency of the proposed model. We present the confusion matrices for route classification using the BLE-MMDTW and MMDTW method in Fig. 6(a) \& Fig. 6(b), respectively. The proposed BLE-MMDTW model provides a reasonable classification accuracy of $85 \%$ and outperforms the MMDTW method that provides a classification accuracy of $76.67 \%$. However, the MMDTW method performs well for route classification when compared to classification of micro-activities since the observed trajectories have a more unique magnetic signature for trajectories when compared to

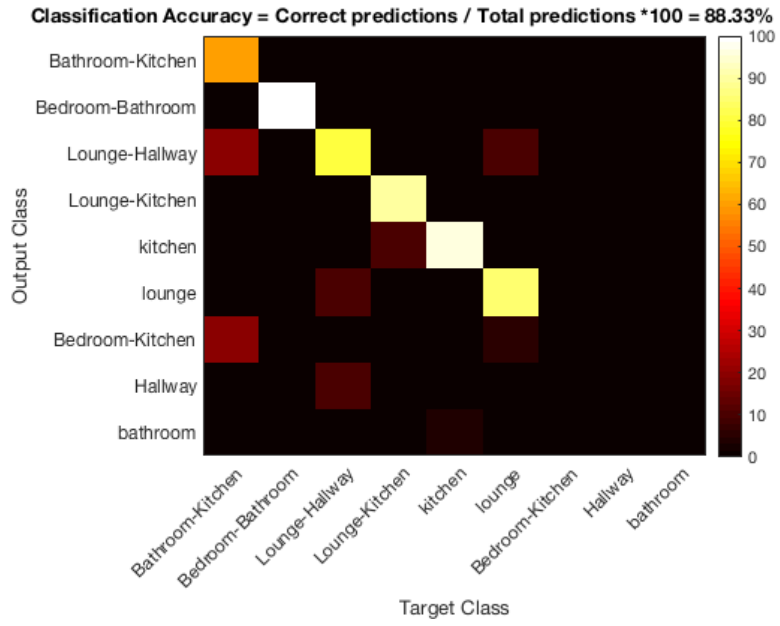

(a) BLE-MMDTW Method

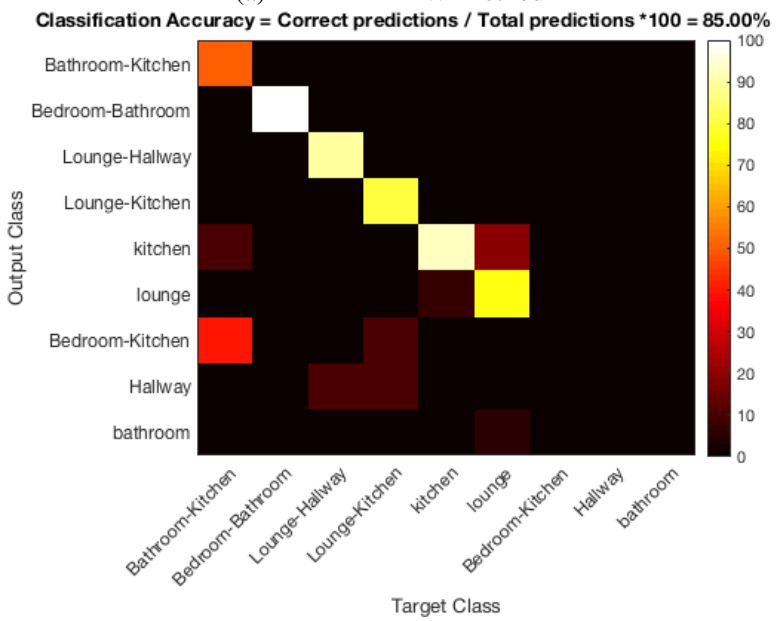

(b) MMDTW Method

Fig. 8. Recognition confusion matrix for zone-based classification of walking routes

stationary points. In the case of trajectories, we used Fréchet distance as the performance metric to measure the similarity between the ground truth and the predicted outcome [28]. It takes into account the location and ordering of the distance coordinates along a trajectory, which makes it a suitable distance metric when comparing 2 different walking routes of different lengths. The Fréchet distance measure can be mathematically represented by $\mathrm{Eq}(8)$.

$$
d_{F}(P, Q)=\min _{\substack{\alpha[0,1] \rightarrow[0, N] \\ \beta[0,1] \rightarrow[0, M]}}\left\{\max _{t \in[0,1]} d(P(\alpha(t)), Q(\beta(t))\}\right.
$$

where $P$ and $Q$ are the actual and predicted trajectories of lengths $N$ and $M$, respectively and $d$ is the distance function. The co-ordinates $\alpha(\mathrm{t})$ in $P$ and $\beta(\mathrm{t})$ in $Q$ range over continuous and increasing functions with $\alpha(0)=0, \alpha(1)=N, \beta(0)=$ 0 and $\beta(1)=M$. The Fréchet distance measures for the selected routes using the BLE-MMDTW (with step-detector) and MMDTW methods are shown in Fig. 7.

For zone-based classification, the BLE-MMDTW and MMDTW methods provide a classification accuracy of $88.33 \%$ and $85 \%$, respectively. Their respective confusion ma- 


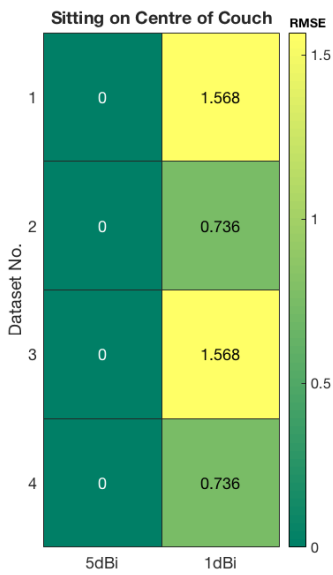

(a) Sitting on Centre of Couch

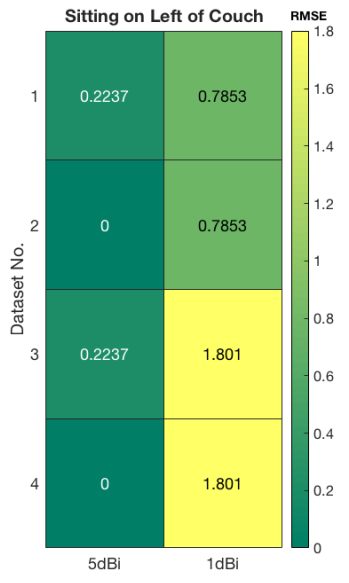

(b) Sitting on Left of Couch

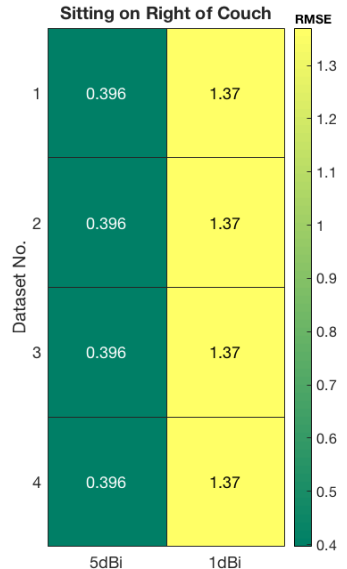

(c) Sitting on Right of Couch

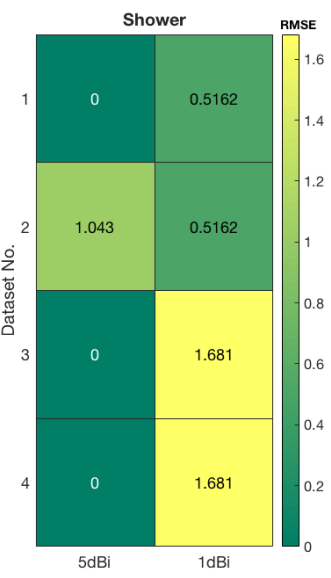

(d) Using the Shower

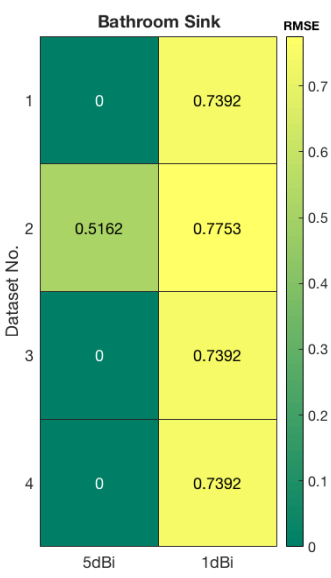

(e) Using the Bathroom Sink

Fig. 9. Performance comparison of RMSE of individual micro-activities in Trial home-2

trices for the associated intra-zone and inter-zone classification of routes are shown in Fig. 8(a) \& Fig. 8(b).

\section{Selection of Time Window Length for Segmentation of Incoming Data in Trial home-1}

A sliding window approach was used for segmentation of incoming wearable sensor data for near real-time prediction of micro-activities and walking routes in Trial home-1. Based on the sampling rate and the length of the trajectories to be measured, a suitable time window, $T_{w}$ length needs to be selected such that the segmented data contains sufficient information to output a micro-activity or trajectory. In order to test the system responsivity, the classification accuracy of routes for three different time windows of length $T_{w 1}=8 \mathrm{~s}$ ,$T_{w 2}=12 \mathrm{~s}$ and $T_{w 3}=15 \mathrm{~s}$ were computed. When $T_{w 1}$ and $T_{w 2}$ are the chosen parameters, a classification accuracy of $66.67 \%$ and $77.67 \%$ were obtained, respectively. We chose $T_{w 3}=15 \mathrm{~s}$ for which a maximum classification accuracy of $85 \%$ is achieved.

\section{E. Performance Assessment in Trial home-2 with Reduced Receivers}

A different comparative analysis was conducted in Trial home-2 to study the impact of reducing the number of receivers and the effect of higher receiver antenna gain on the final outcome using the proposed algorithm. As described earlier, we chose to deploy five Raspberry-Pi receivers equipped with external antennas $(5 \mathrm{dBi}$ and $1 \mathrm{dBi})$ in the slightly larger second trial home to test the robustness of the hybrid approach with lesser number of receivers. As part of this study, we selected five micro-activities and five routes for which four test sample data of each of them are collected on different days spread over a week.

a) Experimental Results for Micro-activities: RMSE is used as the metric to assess the accuracy of the predicted micro-activities. The five micro-activities selected for analysis were: 1) Sitting on Centre of Couch, 2) Sitting on Left of Couch, 3) Sitting on Right of Couch, 4) Using the Shower, 5) Using the Bathroom Sink. Fig. 9 illustrates the RMSE for these micro-activities when the experimental study was conducted separately with receivers fitted with $5 \mathrm{dBi}$ and $1 \mathrm{dBi}$ antennas respectively. The results indicate that the RMSE of predicted activities is a lot smaller when the receivers are fitted with $5 \mathrm{dBi}$ antennas than with $1 \mathrm{dBi}$ antennas. This is because an increase in antenna gain improves the range of the Raspberry$P i$ receivers such that they can detect the beacon from a greater distance [27]. Hence sufficient accuracy is still maintained with reduced number of receivers when equipped with high gain antennas. However, accuracy is compromised with lowgain antennas due to reduced coverage as only five receivers are used in Trial home-2 compared to eight in Trial home1. In cases where high gain antennas are used, the predicted outcome is precise in most cases for activities $\{1,2,4,5\}$ and in other instances provides sub-meter level precision. Similar to Trial home-1, the locus of the predicted activity for different sitting positions on the couch (Activities $\{1-3\}$ ) using $5 \mathrm{dBi}$ antennas for all test samples was found to be the Couch. We can therefore avoid the use of a pressure sensor and rely solely on the positioning results to find out if the person has been spending some time on the couch. Opting for low gain antennas can also prove useful if the requirement is only to predict the room or zone where the activity is taking place. The average error is around a meter when low gain antennas are used.

b) Experimental Results for Walking Routes: The walking routes selected for analysis in Trial home-2 were 1) Bathroom to Kitchen Fridge, 2) Kitchen Fridge to Bathroom, 3) Kitchen Fridge to Sink, 4) Couch to Front Door, 5) Couch No.2 to Front Door. A comparative analysis was performed for walking routes when the receivers were fitted with $5 \mathrm{dBi}$ and $1 \mathrm{dBi}$ antennas, respectively. Similar to Trial home-1, we used Fréchet distance as the performance metric to measure the similarity between the actual and predicted results. The Fréchet 


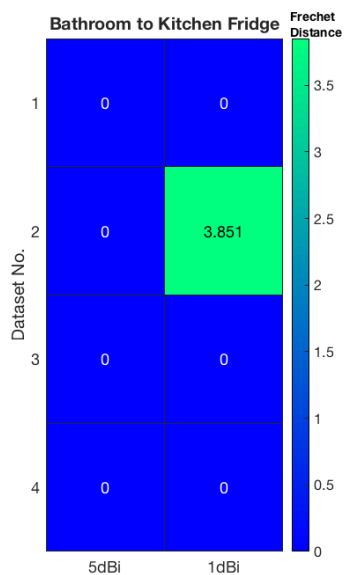

(a) Bathroom to KitchenFridge

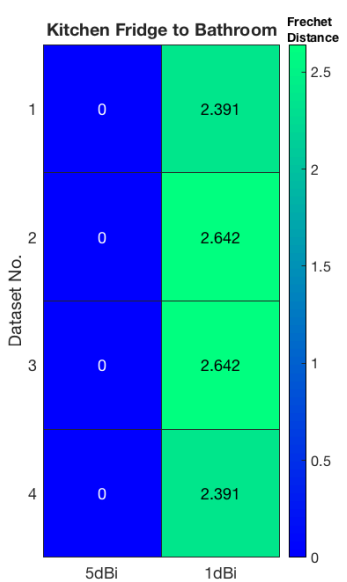

(b) KitchenFridge to Bathroom

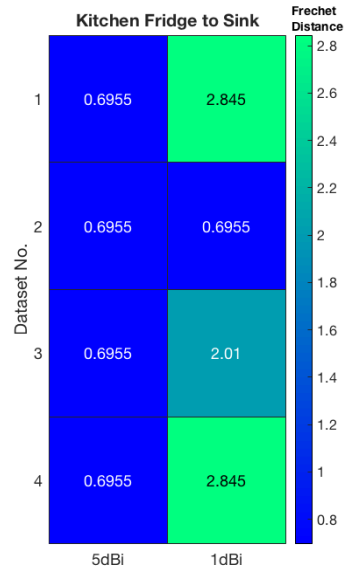

(c) Kitchen Fridge to Sink

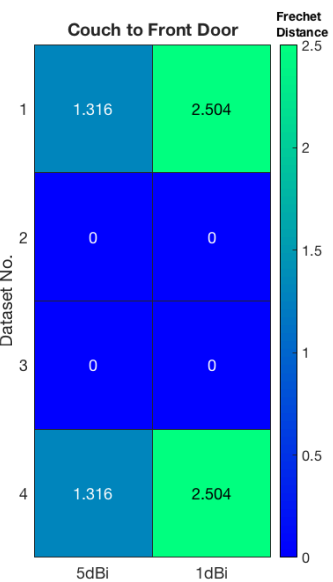

(d) Couch to Front Door

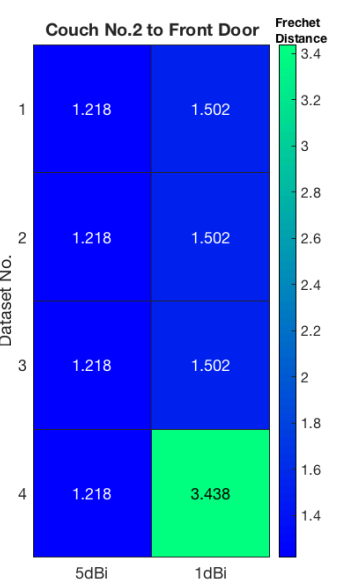

(e) Couch No.2 to Front Door

Fig. 10. Performance comparison of walking routes using Fréchet Distance in Trial home-2

distance results of the selected five routes are illustrated in Fig. 10. The predicted outcome for routes 1 and 2 were precise when using $5 \mathrm{dBi}$ antennas for each of the four test datasets as seen in Fig. 10(a) and Fig. 10(b). Overall, the Fréchet distance between the target route and the predicted route when using high gain $5 \mathrm{dBi}$ antennas is considerably lesser when compared to using low gain $1 \mathrm{dBi}$ antennas for all the five routes indicating high similarity between the predicted route and the actual route. In comparision, the Fréchet distances while using $1 \mathrm{dBi}$ antennas are slightly on the higher side as the overall coverage by the receivers is reduced across various rooms in the flat. Thus, it can be deduced that increasing the receiver antenna gain raises the likelihood of improvement and helps reduce the number of Raspberry-Pis deployed in the test environment while maintaining sufficient accuracy.

\section{F. Case Study Evaluation}

A case study evaluation was conducted in the two test apartments to evaluate the performance of the proposed method employing the BLE-MMDTW approach against the solo use of MFV fingerprinting. This was determined when the user was moving inside the apartment performing a series of random activities that reflect regular human day-to-day behavior. The test results of Trial home-1 and Trial home-2 (using $5 \mathrm{dBi}$ antennas) are highlighted in Fig. 11 and Fig. 12, respectively. The positions or routes specified in both the figures are the results of each method, which are in the order of the activities performed by the user. The resulting output from the case studies prove that the predicted routes and positions obtained by the proposed method are much closer to the actual results as compared to using only MFV sequences for location estimation. Furthermore, the results indicate that the independent use of the MFV fingerprinting method is ineffective and therefore contributes to a high mismatch rate.

\section{Discussion}

The need to design a reliable context based location aware system for an increasingly dynamic and complex domestic environment is crucial as they form the basis for a number of remote home healthcare applications. Sequence-based inputs require specialized classifier algorithms as they are different from other supervised learning problems. The accuracy of the proposed algorithm is not compared against well-known machine learning classifiers in this paper as most of these classifiers are not suitable for sequence classification problems. Recurrent Neural Networks (RNN) using Long Short-Term Memory (LSTM) networks are one of the few state-of-the-art techniques known for sequence classification. However, the size of the radio map required for training LSTM networks needs to be large to achieve good prediction accuracy. Hence, a novel sequence based algorithm that requires less training data has been developed. Table II shows a comparison of our proposed system against recent works that have used location estimation techniques as a supporting or standalone system for activity monitoring. Most of these systems are designed only to achieve room-level accuracy [13][18][19]. Other works that consider a higher number of activities, suffer in accuracy due to the complexity involved in a multi-activity classification problem (10 or more classes) [15][20]. Location estimation techniques are used in combination with other sensing methods in both these works. In this study, we have used indoor positioning techniques as a standalone system to provide more fine-grained positioning rather than room-level accuracy and to classify multiple activities. Furthermore, our study also considers the user trajectory, which is crucial for continuous monitoring applications. Despite the complexity involved, we have managed to achieve reasonable accuracy for both stationary positions and walking routes.

The results from Section VI demonstrate that the hybrid BLE-MMDTW method with the inclusion of step detector information outperforms the individual use of MMDTW method in all the test case scenarios for micro-activities and walking routes. The findings are more conclusive since they were assessed in a home environment with strong NLOS conditions. Overall, the performance assessment and the case study results 


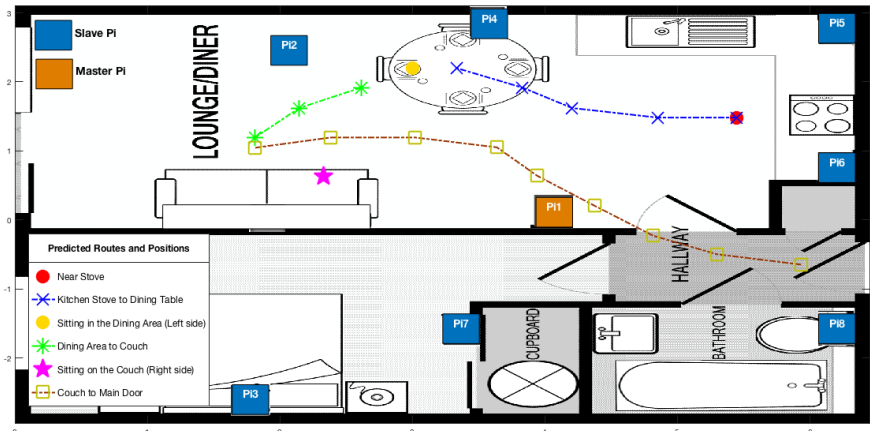

(a) Actual Walking Route

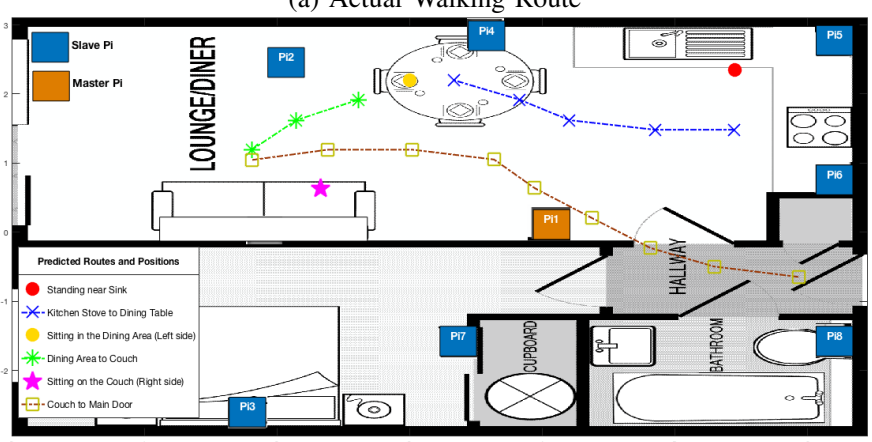

(b) Predicted Walking Route (Using Beacon RSSI + MFV Fingerprinting

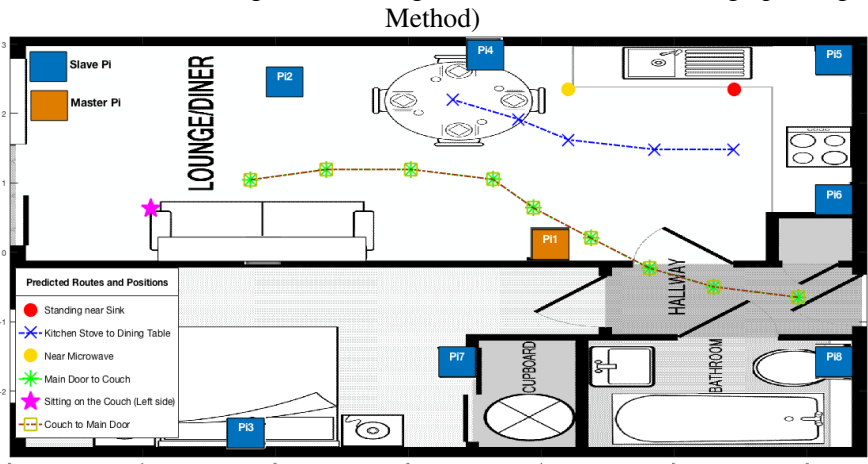

(c) Predicted Walking Route (Using only MFV Fingerprinting Method)

Fig. 11. Case Study Illustration when user performs the following activities in Trial home-1 [Stands near the Stove - Moves to Dining Table from Stove - Sits in left side of the Dining area - Moves to the Couch - Sits on the right side of the Couch - Moves towards the Main Door]

corroborate the hypothesis that the fusion of BLE and MFV fingerprinting with added information from inertial sensors complement each other to accomplish low-level recognition of multiple activities and user trajectory prediction in a smart home environment. Furthermore, the decision to evaluate $1 \mathrm{dBi}$ and $5 \mathrm{dBi}$ interchangeable external antennas in Trial home-2 was to determine the level of localization accuracy with fewer receivers using the proposed algorithm. Experimental results indicate that adequate accuracy can still be maintained with a reduced infrastructure, by deploying receivers with higher gain antennas. Besides, the data collection approach used in this study along with radio map construction for sequencebased inputs and trajectory measurements can be extended to other location estimation technologies (Eg: LiDAR, Radar, mmWave). Our future research direction will concentrate on extending the proposed methodology for implementation in

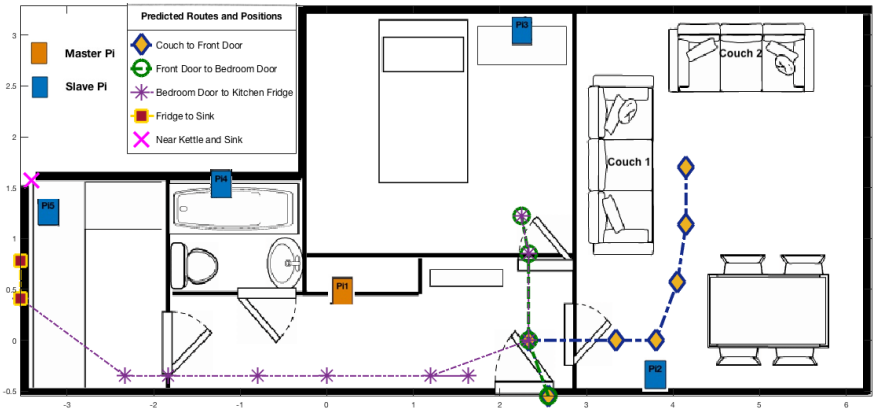

(a) Actual Walking Route

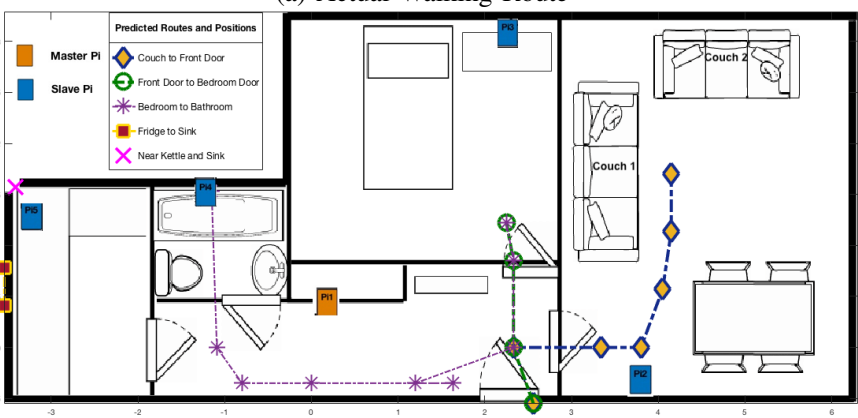

(b) Predicted Walking Route (Using Beacon RSSI + MFV Fingerprinting

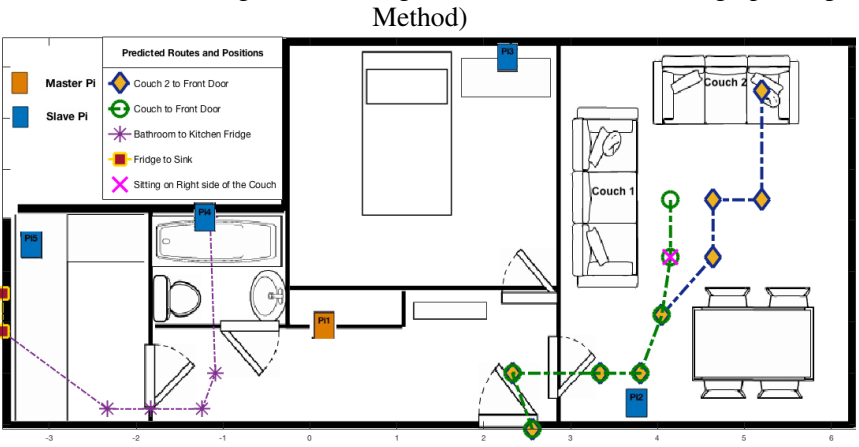

(c) Predicted Walking Route (Using only MFV Fingerprinting Method)

Fig. 12. Case Study Illustration when user performs the following activities in Trial home-2 [Moves from the Couch towards the front door - Walks towards the bedroom door - Moves to the kitchen fridge from the bedroom door - Turns and moves towards the sink - Stands near the Kettle and Sink]

different types of home and testing with individuals who belong to different age groups making use of the same training database. Work will also be done in testing the overall performance efficiency for recognition of complex activities using low-level context obtained using the proposed method in this paper.

\section{CONCLUSION}

In this work, a novel algorithm has been developed for low-level micro-activity recognition and prediction of walking routes using wearable sensing. The implementation employs an inverse beacon fingerprinting scheme coupled with inertial sensors to narrow down the magnetic field vector matching space. The suggested approach helps in overcoming the shortcomings of beacon signal stability and mismatch issues in magnetic field fingerprinting. An overall improvement in prediction accuracy is made possible by amalgamating the results of both techniques. Furthermore, a context-oriented, 
TABLE II

COMPARISON OF ACTIVITY MONITORING SYSTEMS USING LOCATION ESTIMATION TECHNIQUES

\begin{tabular}{|c|c|c|c|c|c|c|c|}
\hline Reference & $\begin{array}{c}\text { Connection } \\
\text { Type }\end{array}$ & Method Employed & Devices Used & Cost & $\begin{array}{l}\text { Trajectory } \\
\text { Prediction }\end{array}$ & Avg. Accuracy \& Range & No. of Classes \\
\hline$[13]$ & Wi-Fi & Fingerprinting & Smartphone & Low & No & $89 \%$; Room-level accuracy & 8 activity classes \\
\hline$[20]$ & $\begin{array}{l}\text { Bluetooth; } \\
\text { ZigBee }\end{array}$ & Random Forest classifier & $\begin{array}{c}\text { Power meters; current } \\
\text { transformers; Ultrasonic sensor }\end{array}$ & Medium & No & $79.39 \%$ & $\begin{array}{l}10 \text { class high-level } \\
\text { activities }\end{array}$ \\
\hline$[18]$ & BLE & $\begin{array}{l}\text { Smoothing, machine learning } \\
\text { algorithms with fingerprinting }\end{array}$ & $\begin{array}{l}\text { Wearable beacon; fixed } \\
\text { raspberry-pis }\end{array}$ & Low & No & $\begin{array}{l}\text { Precision: } 84.2 \% \text {; F-measure: } \\
\text { 80.9\%; Room-level accuracy }\end{array}$ & 6 ADL classes \\
\hline [19] & BLE & Fingerprinting & Fixed beacons; smartphone & Low & No & $\begin{array}{l}93 \% \text { (room estimation; } 83 \% \text { (frailty } \\
\text { classification); Room-level accuracy }\end{array}$ & $\begin{array}{l}3 \text { class frailty } \\
\text { classification }\end{array}$ \\
\hline$[15]$ & BLE & Recursive Bayesian approach & $\begin{array}{l}\text { binary sensors, capacitive smart } \\
\text { floor; smart watch with beacon }\end{array}$ & High & No & $68 \%$ & 24 high-level ADL's \\
\hline $\begin{array}{l}\text { Proposed } \\
\text { Method }\end{array}$ & BLE & $\begin{array}{c}\text { Sequence Matching; DTW } \\
\text { Algorithm using WiFi \& MFV } \\
\text { Fingerprinting with stepdetection }\end{array}$ & $\begin{array}{l}\text { Wearabe (beacon, accelerometer, } \\
\text { gyroscope, magnetometer); } \\
\text { Raspberry-Pi receivers }\end{array}$ & Low & Yes & $\begin{array}{l}\text { Micro-activity: } \text { RMSE }=0.55 \mathrm{~m} ; \\
91.87 \% \text { (zone) } ; \text { Routes }=85 \% \\
\quad 88.33 \% \text { (zone) }\end{array}$ & $\begin{array}{l}16 \text { micro-activities; } \\
12 \text { routes (Total: } 40 \\
\text { positions/routes) }\end{array}$ \\
\hline
\end{tabular}

trajectory-based radio map model for location estimation is adopted in this study to provide a realistic scenario for testing that is better suited in setting up an activity recognition system at home. The empirical results demonstrate that the proposed method has high potential in providing centimeterlevel positioning accuracy for micro-activities and a reasonable classification accuracy over $80 \%$ can be achieved for walking routes. The method proposed in this paper provides an accurate and cost-effective solution for monitoring applications within a home environment as it delivers sufficient prediction accuracy on its own without the use of object-based sensing methods. Furthermore, complex ADL recognition is feasible when the suggested method is combined with posture recognition methods or used in ambient sensing environments, instrumented with only the essential sensors required for monitoring.

\section{REFERENCES}

[1] M. E. Mlinac, M. C. Feng, "Assessment of activities of daily living, selfcare, and independence", Archives of Clinical Neuropsychology, Vol. 31, Issue 6, pp.506-516, Aug 2016.

[2] H. Liu, H. Darabi, P. Banerjee, J. Liu, "Survey of wireless indoor positioning techniques and systems", IEEE Trans. Syst. Man Cybern. C: Applications and Reviews, vol. 37, no. 6, pp. 1067-1080, Nov. 2007.

[3] V. Pasku et al., "Magnetic Field Based Positioning Systems." IEEE Comm. Surveys \& Tutorials, Mar 2017.

[4] K. Nguyen, Z. Luo, "Dynamic route prediction with the magnetic field strength for indoor positioning", Int. Journal of Wireless and Mob. Computing,Vol.12 Issue 1, Pages 16-35, Jan 2017.

[5] B. Gozick, K. P. Subbu, R. Dantu, T. Maeshiro, "Magnetic maps for indoor navigation", IEEE Trans. Instrum. Meas., vol. 60, no. 12, pp. 3883-3891, Dec. 2011.

[6] A. Alarifi et al., "Ultra wideband indoor positioning technologies: Analysis and recent advances," Sensors (Basel), vol. 16, no. 5, p. 707, May 2016.

[7] J. Torres-Sospedra, R. Montoliu, G. M. Mendoza-Silva, O. Belmonte, D. Rambla, J. Huerta, "Providing databases for different indoor positioning technologies: Pros and cons of magnetic field and Wi-Fi based positioning," in Proc. Mobile Inf. Syst., vol. 2016, pp. 1-22, Jan. 2016.

[8] X. Zhao, Z. Xiao, A. Markham, N. Trigoni, and Y. Ren, "Does BTLE measure up against WiFi? A comparison of indoor location performance," in Proc. Eur. Wireless 20th Eur. Wireless Conf., pp. 1-6, Barcelona, Spain, 2014

[9] P. Davidson and R. Piche, "A survey of selected indoor positioning methods for smartphones", IEEE Comm. Surveys \& Tutorials, 2016.

[10] Y. Li, Y. Zhuang, H. Lan, P. Zhang, X. Niu, N. El-Sheimy, "Wi-FiAided magnetic matching for indoor navigation with consumer portable devices", Micromachines, vol. 6, no. 6, pp. 747-764, 2015.

[11] E. Wang, M. Wang, Z. Meng, X. Xu. "A Study of WiFi-Aided Magnetic Matching Indoor Positioning Algorithm." Journal of Computer and Comm. 5.03, pp. 91-101, 2017

[12] S. Dernbach, B. Das, N. C. Krishnan, B. L. Thomas, D. J. Cook, "Simple and complex activity recognition through smart phones", Proc. 8th IE, pp. 214-221, Jun. 2012.
[13] J. Torres, O. Belmonte, R. Montoliu, S. Trilles, and A. Calia, "How feasible is WiFi fingerprint-based indoor positioning for in-home monitoring?" in Proc. 12th Int. Conf. Intell. Environ. (IE), Sep. 2016, pp. $68-75$.

[14] M. Philipose et al., "Inferring activities from interactions with objects", IEEE Pervasive Computing 3(4):50- 57, Nov 2004.

[15] A. R. Jimenez and F. Seco, "Event-driven Real-time Location-aware Activity Recognition in AAL Scenarios," in Proc. 12th Int. Conf. on Ubiq. Computing and Amb. Intell., UCAmI 2018, pp. 1-12, 4-7 Dec, 2018; Punta Cana, Dominican Republic.

[16] H. Zhou and H. Hu, "Human motion tracking for rehabilitation - A survey," Biomedical Signal Processing and Control, vol. 3, no.1, pp. $1-18,2008$.

[17] K. Arning et al., "Get that Camera Out of My House!", Conjoint Measurement of Preferences for Video-Based Healthcare Monitoring Systems in Private and Public Places" in Inclusive Smart Cities and e-Health, Springer Int. Publishing, pp. 152-164, 2015.

[18] T. Morita et al., "Beacon-Based Time-Spatial Recognition toward Automatic Daily Care Reporting for Nursing Homes," Journal of Sensors, vol. 2018, Article ID 2625195, pp. 1-18, 2018.

[19] T. Tegou et al., "A Low-Cost Indoor Activity Monitoring System for Detecting Frailty in Older Adults", Sensors, vol.19(3), pp.1-19, Jan 2019.

[20] E. Nakagawa, K. Moriya, H. Suwa, M. Fujimoto, Y. Arakawa, K. Yasumoto, "Toward real-time in-home activity recognition using indoor positioning sensor and power meters", IEEE Int. Conf. on Perv. Comp. and Comm. (PerCom Workshops), pp. 539-544, 2017.

[21] B. Zhou, Q. Li, Q. Mao, W. Tu, "A robust Crowdsourcing-based indoor localisation system", Sensors, vol. 17, no. 4, pp. 864, 2017.

[22] M. Sridharan, J. Bigham, C. Phillips, E. Bodanese, "Collaborative location estimation for confined spaces using magnetic field and inverse beacon positioning", pp. 1-3, IEEE Sensors 2017.

[23] X. Ye et al., "WarpMap: accurate and efficient indoor location by dynamic warping in sequence-type radiomap", In Proc. of the 2016 13th Annual IEEE Int. Conf. on Sensing, Comm., and Networking (SECON), 27-30, pp.1-9, London, UK, Jun 2016.

[24] J.H.An, L.Choi, "Inverse fingerprinting: server side indoor localization with bluetooth low energy", In Proc. of the 27th Annual IEEE Int. Symposium on Personal, Indoor and Mob. Radio Comm. (PIMRC): Mobile and Wireless Networks, 2016.

[25] K. P. Subbu, B. Gozick, R. Dantu, "Indoor localization through dynamic time warping", In Proc. of the Systems, Man, and Cybernetics (SMC), Anchorage, AK, USA, 9-12 October 2011.

[26] Mbientlab, 2018. Product Specification v0.5. [Online]. Available at: https://mbientlab.com/documents/MetaMotionR-PS3.pdf [Accessed 24th September 2018].

[27] M. Sridharan, J. Bigham, P. M. Campbell, E. Bodanese, "Evaluation of Factors Affecting Inverse Beacon Fingerprinting Using Route Prediction Algorithm", IEEE WCNC 2019 [In press].

[28] H.Alt, M.Godau, "Computing the Fréchet distance between two polyg onal curves", Int. J. Comput. Geom. Appl., Vol.5(1-2), 75-91, 1995. 